\title{
Trends in the portion size of savoury snack intakes in Irish adults during 2001 and 2011
}

\author{
S. O’Brien ${ }^{1}$, J. Lyons ${ }^{2}$, B. A. McNulty ${ }^{1}$, J. Walton ${ }^{2}$, A. Flynn ${ }^{2}$, M. J. Gibney ${ }^{1}$, A. P. Nugent ${ }^{1}$, \\ E. R. Gibney ${ }^{1}$ and M. B. E. Livingstone ${ }^{3}$ \\ ${ }^{1}$ Institute of Food and Health, University College Dublin, Belfield, Dublin 4, Republic of Ireland, ${ }^{2}$ School of Food and \\ Nutritional Sciences, University College Cork, Republic of Ireland and ${ }^{3}$ Northern Ireland Centre for Food and Health, \\ University of Ulster, Coleraine, UK
}

Larger portion sizes of foods and increased "snacking" have been linked to an overall increase in energy intake ${ }^{(1)}$. In terms of snacking, recent U.S. research cites "salty snacks" as the most influential snack in the increase of overall energy intake from 18-24\% ${ }^{(2)}$. Trends in the portion sizes of foods such as savoury snacks have not previously been investigated. Food portion sizes ( $\mathrm{g}$ ), defined as the weight of the food consumed per eating occasion, were estimated using data from the North/South Ireland Food Consumption Survey (NSIFCS) and the National Adult Nutrition Survey (NANS). The NSIFCS collected data from 1274 adults (18-64 years) between 1997-1999 using a 7-d semi-weighed food record, while the NANS used a 4-day semi-weighed food record to collect data from 1379 adults (2009-2010). Total savoury snacks and the most frequently consumed sub-groups of savoury snacks, as well as total nuts and seeds were selected for analysis, accounting for 2803 of 214,563 eating occasions in NSIFCS and 98,466 in NANS. Of the savoury snacks selected, the majority ( $87 \%$ and $92 \%$ in NSIFCS and NANS, respectively) of food weights were quantified by weighed data. Data are presented for consumers only $(51.3 \%$ and $36.9 \%$ of NSIFCS and NANS, respectively) at a total population level.

\begin{tabular}{|c|c|c|c|c|c|c|c|c|c|}
\hline \multirow[b]{2}{*}{ Food Type } & \multicolumn{4}{|c|}{ NSIFCS } & \multicolumn{4}{|c|}{ NANS } & \multirow[b]{2}{*}{$P$} \\
\hline & $n$ & $\mathbf{M}^{\wedge}$ & $25^{\text {th }}$ & $75^{\text {th }}$ & $n$ & $\mathbf{M}^{\wedge}$ & $25^{\text {th }}$ & $75^{\text {th }}$ & \\
\hline Savoury snacks*, total & 1703 & 30 & 25 & 32 & 1000 & 26 & 25 & 37 & 0.001 \\
\hline Crisps, potato & 1321 & 30 & 27 & 31 & 611 & 25 & 25 & 37 & 0.040 \\
\hline Crisps, other i.e. corn snacks & 219 & 25 & 17 & 30 & 154 & 24 & 19 & 26 & NS \\
\hline Popcorn, all & 77 & 30 & 30 & 38 & 147 & 30 & 28 & 50 & NS \\
\hline Nuts, all & 239 & 25 & 11 & 30 & 269 & 24 & 10 & 50 & NS \\
\hline Seeds, all & 52 & 10 & 6 & 12 & 290 & 5 & 3 & 10 & 0.001 \\
\hline
\end{tabular}

*denotes all types of crisps (potato, maize corn snacks), popcorn, pretzels etc. (excludes nuts/seeds), $n$ denotes eating occasions, $\mathrm{M}^{\wedge}$ denotes median portion weight ( $\mathrm{g}$ ),

bold denotes statistically different between surveys with $p<0.05$ (Mann-Whitney U-tests using the Bonferroni correction), NS denotes not statistically different.

A significant decrease in the median portion weights of all savoury snacks and seeds was seen over the ten year period. When split into sub-groups, this trend remained in potato crisps only. Potato crisps account for much of the total eating occasions of savoury snacks, and include potato crisps purchased in a variety of packaging sizes (multi-pack bags, "share" bags etc). Previous research from the U.K. (3) found crisps sold as a multi-pack to have a median portion size of $25 \mathrm{~g}$, while those sold separately tended to have larger median portion weights, suggesting that the variety in available packaging sizes may have an effect on consumption. Interestingly, the number of eating occasions of seeds increased almost six-fold, despite the latter survey consisting of shorter (4-day) food records, but the median portion size significantly decreased. In conclusion, the median portion sizes of a range of savoury snacks commonly consumed in Ireland are, in general, decreasing.

This material is based upon works supported by: Safefood, the Food Safety Promotion Board, under Grant No. 07-2010.

1. Piernas C \& Popkin BM (2011) American Journal of Clinical Nutrition 4, 1324-1332.

2. Piernas C \& Popkin BM (2010) Journal of Nutrition 140(2): 325-332.

3. Church S (2008) Report to the Food Standards Agency. 